\section{Account for the 'dark matter' of biology}

Philip Ball correctly opines of DNA that "we should lift some of the awesome responsibility for life's complexity from its shoulders" (Nature 496, 419-420; 2013). In addition to the '-omes' he lines up for this responsibility, other components that are vital to all life forms are the metabolome (small-molecule intermediates), the lipidome (including cellular membranes) and the glycome (massive arrays of sugar chains on cell surfaces and extracellular compartments).

We now know that these other '-omes' mediate much of the diversity and complexity found in natural biological systems. Ball makes an apt analogy to the 1998 discovery that the expansion of the Universe is accelerating, which forced cosmologists to think beyond the standard model and realize that dark matter and dark energy actually dominate many key processes.

Likewise, it is time for more biologists to venture beyond the standard model of biology (involving DNA, RNA and proteins) that has served us so well, and take into account the 'dark matter' of the biological universe - as well as the physical, biological, social and cultural environment.

Ajit Varki University of California, San Diego, La Jolla, USA. a1varki@ucsd.edu

\section{Getting the word out on biosphere crisis}

A landmark statement released last week, formulated from a review published in Nature last year, illustrates how effectively and rapidly bridges can be built between science and society.

The review suggested that human influence may be forcing the global ecosystem towards a rapid, irreversible, planetary-scale shift (A. D. Barnosky et al. Nature 486, 52-58; 2012). California's governor, Jerry Brown, invited the authors to draw their findings to the attention of policy-makers, industry and the public. An international group of 16 scientists spent the next few months developing the statement and circulating it to the globalchange scientific community.

The resulting statement 'Maintaining humanity's life support systems in the 21 st century' (see go.nature.com/ prudoq) - is endorsed by more than 500 global-change researchers whose work spans every continent. It warns that unless decisive countermeasures are put into place immediately, climate change, loss of ecological diversity, extinctions environmental contamination, human population growth and overconsumption of resources will degrade our quality of life within a few decades.

The statement is already catalysing interactions between governments, universities, business leaders and scientists to educate local constituencies about global issues and to stimulate solutions.

Elizabeth A. Hadly Stanford University, California, USA. hadly@stanford.edu

${ }^{*}$ On behalf of 4 co-signatories. See go.nature.com/sgrxwo for full list.

\section{Boost to translational medicine in Europe}

Next week will mark the launch of the European Infrastructure for Translational Medicine (EATRIS; see go.nature. com/3lisfs). EATRIS aims to help clients (industry, small enterprises, funding organizations and academic institutions) to translate their biomedical discoveries efficiently into preventive, diagnostic or therapeutic products up to the point of clinical proof of concept.

More than 60 leading

translational-research centres will make their facilities and expertise available to researchers and industry across Europe, through a single point of entry, bringing together basic scientists and clinicians, as well as public and private partners. These centres may be specialists in, for example, advanced therapies, imaging or biomarkers.

EATRIS will minimize development costs and risks by matching expertise and optimizing the use of academic know-how, infrastructure and patient groups. Quality control, intellectual property and legal frameworks will all be standardized. Rapidly assembled multidisciplinary teams will address each project's specific regulatory and clinical needs.

These measures should also attract much-needed attention to rare and orphan diseases.

Giovanni Migliaccio, Frank H. de Man, Anton E. Ussi EATRIS Coordination and Support, Amsterdam, the Netherlands.

giovannimigliaccio@eatris.eu

\section{Don't oversimplify psychiatric disorders}

Psychiatric diagnosis is indeed imperfect, subjective and not based on pathophysiology or causation (Nature 496, 416-418; 2013). But quests for biological markers and dimensional approaches, such as the Research Domain Criteria project mentioned, are unlikely to be clinically appropriate solutions because they run the risk of oversimplifying complex psychiatric illnesses and introducing predicative logic.

For instance, people with post-traumatic stress disorder (PTSD) or psychosis may show similar abnormalities of frontallobe function on a magnetic resonance imaging scan, just as those with anxiety or psychosis may show similar overactivation of the amygdala in the brain. But the underlying reasons for these superficial similarities are different, and so are the treatments. For example, lithium is not an effective treatment for psychosis, anxiety or PTSD, but it is near-curative for some people who meet current diagnostic criteria for bipolar disorder.

The genetics of some conditions may overlap (for example, schizophrenia, autism and bipolar disorder), as do the genetics of, say, multiple sclerosis and Crohn's disease. However, these are distinct clinical conditions that require different interventions.

Caution and healthy scepticism are essential before embracing fashionable trends to revise psychiatric diagnosis.

Daniel R. Weinberger Johns Hopkins University School of Medicine, Baltimore, Maryland, USA.

drweinberger@libd.org

\section{Communications need NIH funding}

The US Congress launched an enquiry into all 'public relations' expenditure by the US National Institutes of Health (NIH) following coverage in The Cancer Letter of spending by the National Cancer Institute (NCI) Office of Communications and Education (OCE; see Nature $495,142 ; 2013)$. In our view, the criticisms devalue OCE activities and risk diverting attention away from the budget cuts that reduced the NIH's capacity to fund biomedical research.

Critics should note that several OCE activities are mandated or requested by Congress, and that OCE expenditure for the last fiscal year is less than $1 \%$ of the NCI's, so cutting this further will barely affect the NCI's researchfunding capacity.

Communications are an important part of the NIH's mission. The NIH can only benefit from making NCI services and discoveries more accessible to cancer patients, physicians, researchers and the public.

Judith S. Bond, Bethany Drehman Federation of American Societies for Experimental Biology, Bethesda, Maryland, USA. bdrehman@faseb.org 